\title{
HIDROGEOFÍSICA DE LA CUENCA DEL RÍO SAN ANTONIO, EL SALVADOR: APLICACIÓN AL MODELO CONCEPTUAL Y VULNERABILIDAD INTRÍNSECA
}

\author{
Laura Gil Urrutia ${ }^{1} \&$ Mario E. Arias Salguero ${ }^{2 *}$ \\ ${ }^{1}$ Posgrado Centroamericano en Geología, Apdo. 214-2060 \\ Universidad de Costa Rica, San José \\ ${ }^{2 *}$ Escuela Centroamericana de Geología, Apdo. 214-2060 \\ Universidad de Costa Rica, San José \\ *Autor para contacto: marioa@geologia.ucr.ac.cr
}

(Recibido: 24/10/06; aceptado: 30/01/07)

\begin{abstract}
The hydrogeological conceptual model of the river San Antonio basin contemplates the existence of two aquifers: one in the Quaternary piroclastic deposits and other in Quaternary fractured lavas. It has been interpreted from the hydrogeophysical interpretation of 55 vertical electrical soundings, correlated with the information of the static level of 21 dug wells, lithology of 21 drilled wells, and location of 21 springs and San Antonio river differential gaugings. The first aquifer is shallow, unconfined and is exploited for domestic uses. The second one is mainly unconfined and in some sectors is semiconfined, and exploited by drilled wells for the public supply of the municipality of Nejapa. From the point of view of intrinsic vulnerability, it was determined that $20 \%$ of the analyzed sector of the aquifer in the Quaternary piroclastic deposits, presents high vulnerability.
\end{abstract}

Keywords: Hydrogeophysics, intrinsic vulnerability, hydrogeological model, San Antonio's basin, El Salvador.

RESUMEN: A partir de la interpretación hidrogeofísica de 55 sondeos eléctricos verticales, correlacionada con la información de los registros de nivel estático de 21 pozos excavados, litología de 12 pozos perforados, localización de 21 manantiales y aforos diferenciales del río, se establece el modelo conceptual hidrogeológico de la cuenca del río San Antonio, el cual contempla la existencia de dos acuíferos: uno en depósitos piroclásticos del Cuaternario y otro en lavas fracturadas del Cuaternario. El primero es somero, libre y actualmente es aprovechado para uso doméstico a partir de pozos excavados artesanalmente. El segundo es principalmente libre y en algunos sectores es semiconfinado, explotado por pozos perforados para el abastecimiento público del municipio de Nejapa. Desde el punto de vista de vulnerabilidad intrínseca, se determinó que el $20 \%$ del sector analizado del acuífero en depósitos piroclásticos del Cuaternario, presenta vulnerabilidad alta.

Palabras clave: Hidrogeofísica, vulnerabilidad intrínseca, modelo hidrogeológico, cuenca río San Antonio, El Salvador.

URRUTIA, L.G. \& ARIAS, M.E., 2006: Hidrogeofísica de la cuenca del río San Antonio, El Salvador: Aplicación al modelo conceptual y vulnerabilidad intrínseca.- Rev. Geol. Amér. Central, 34-35: 121-129. 


\section{INTRODUCCIÓN}

El término hidrogeofísica es una contracción de la aplicación de la geofísica de prospección con fines hidrogeológicos. Contempla por tanto, la estimación de algunos parámetros y el monitoreo de varios procesos importantes en los estudios hidrogeológicos, con el fin de determinar y cuantificar el recurso agua, comprender el sistema acuífero y brindar herramientas para la evaluación, y en algunos casos, la remediación de zonas contaminadas. Diferentes problemas hidrogeológicos pueden tener solución con la prospección geofísica (Arias, 2002), entre ellos: la determinación de la geometría del acuífero, su caracterización en función de la litología, conocimiento de varios parámetros hidráulicos y la protección de acuíferos superficiales.

La temática hidrogeofísica es objeto cada vez de mayor interés, tanto en artículos, síntesis y sesiones especiales de diferentes conferencias y asociaciones internacionales, entre ellas: International Association of Hydrological Sciences (IAHS), American Geophysical Union (AGU), European Geosciences Union (EGU), European Association of Geoscientists and Engineers (AEGE) y en los simposios de: Application of Geophysics to Engineering and Environmental Problems (SAGEEP) y de la Society of Exploration Geophysicists (SEG).

En El Salvador existen varios informes técnicos realizados por las instituciones encargadas del abastecimiento público, cuyo objetivo es utilizar la prospección geoeléctrica y en especial los sondeos eléctricos verticales, para la localización de pozos, siendo el más significativo el desarrollado por Arévalo (2005), quien presenta un estudio detallado del acuífero Opico-Quezaltepeque, en el cual, la prospección geofísica le permitió evaluar la vulnerabilidad intrínseca de dicho acuífero.

\section{LA CUENCA DEL RÍO SAN ANTONIO}

Se ubica en el municipio de Nejapa, departamento de San Salvador (Fig. 1). El área de la cuenca es de 57,6 km²; sus límites son al norte con el cerro Ojo de Agua y loma el Quebracho, al oeste con loma Las Mesas, al sur con el volcán de San

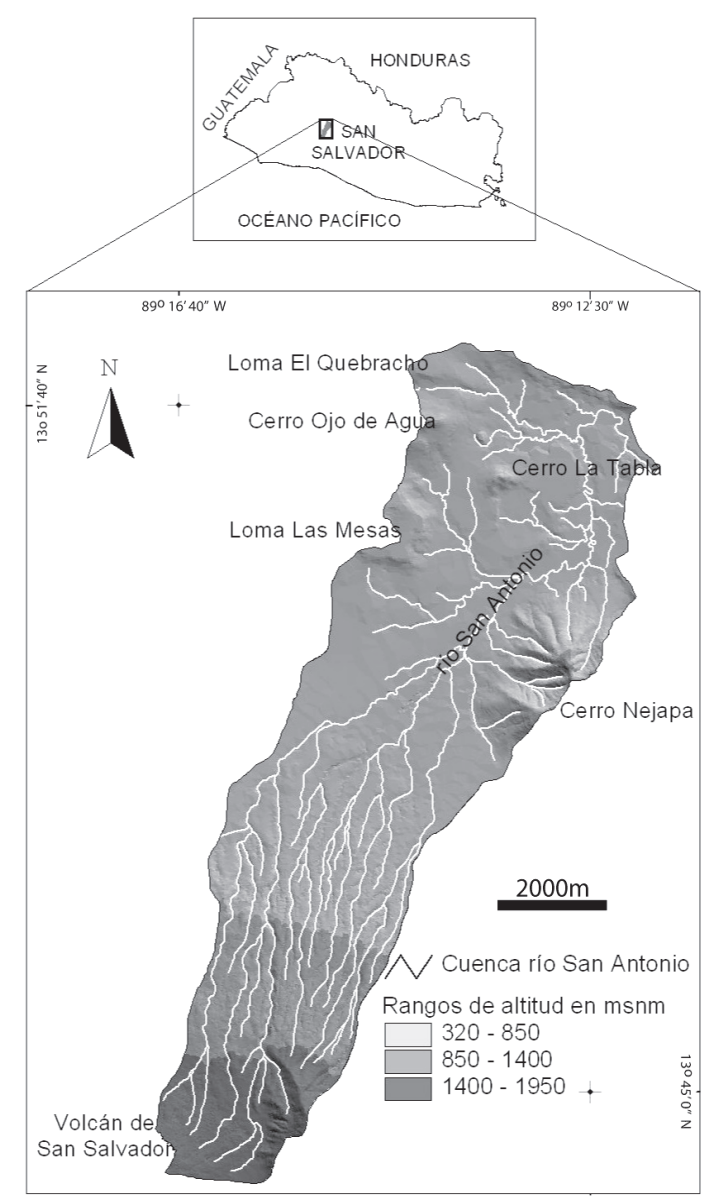

Fig.1: Localización de la zona de estudio.

Salvador, y al este con el cerro de Nejapa. La elevación mayor está en el volcán de San Salvador (1960 m s.n.m.) y la menor a 350 m s.n.m., que corresponde a la desembocadura del río San Antonio en el río Acelhuate.

Geomorfológicamente hay varias estructuras de interés, destacándose el volcán San Salvador y el cerro Nejapa o Champantepeque, los cuales condicionan el patrón de drenaje de esta cuenca, que varía de tipo radial en la zonas altas hasta subdendríico en la zonas bajas y planas. El río San Antonio, nace a una elevación de 450 m s.n.m. en la falda norte del volcán San Salvador.

Wiesemann (1975), establece tres formaciones estratigráficas que afloran en esta cuenca, las cuales se describen de la más antigua a la más reciente (Fig. 2): 


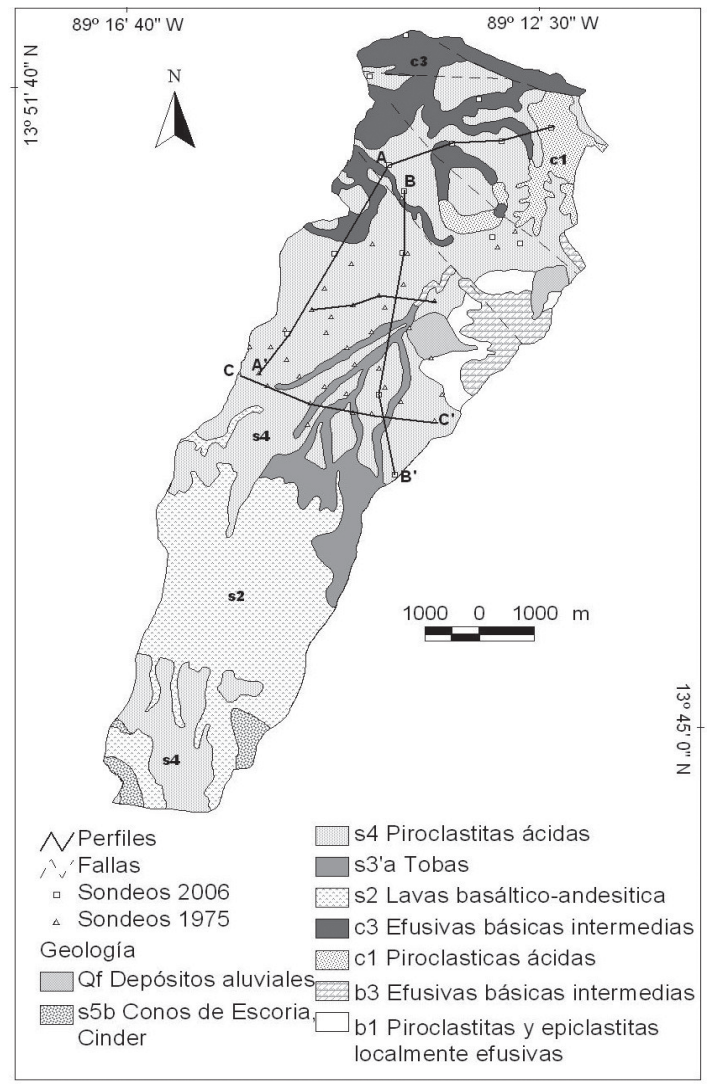

Fig. 2: Mapa geológico y localización de sondeos y perfiles geoeléctricos.

Formación Bálsamo: son rocas tipo ácido en las partes bajas e intermedias y básicas en la parte superior de la formación, pertenecientes al Plioceno, y está conformada por dos miembros denominados b1 y b3. Miembro b1: constituido por epiclastitas volcánicas, piroclastitas e ignimbritas; con lapilli de pómez y limo rojo. Se encuentran además intercalaciones de lavas efusivas básicasintermedias. Miembro b3: constituido únicamente por rocas efusivas básicas-intermedias.

Formación Cuscatlán: se describe como una secuencia de rocas sedimentarias y volcánicas del Plio-Pleistoceno. Los miembros de esta formación son el c1 y el c3. Miembro c1: formado por tobas de lapilli con pómez y fragmentos líticos de lavas dacíticas vítreas y andesitas, inmersas en una matriz de cenizas finas no estratificadas. Miembro c3: compuesto por rocas volcánicas de tipo andesítica y basáltica. Baxter (1984), asocia los productos de este último miembro como resultado de un estratovolcán erosionado.

Formación San Salvador: compuesta por una secuencia de piroclastitas ácidas y efusivas ácidas-básicas intercaladas. Su edad es Pleistoceno Superior a Holoceno. El 80\% del área de estudio está cubierto por materiales pertenecientes a esta formación y los miembros aflorantes son: s2, s3'a, s4, s5'b y Qf. El miembro s2 está compuesto por una secuencia de coladas lávicas andesítico-basálticas, tanto en forma lajeada como escoriácea y presenta piroclastos intercalados. El miembro s3'a está constituido por una secuencia de piroclastitas ácidas y epiclastitas subordinadas, denominadas comúnmente "Tobas color café", las cuales en las cercanías del volcán de San Salvador presentan intercalaciones de escoria negra y capas de suelos desarrollados en diferentes horizontes. El miembro s4 está formado por una secuencia de piroclastitas ácidas, lapilli, pómez y cenizas volcánicas y es denominado comúnmente como "Tierra Blanca”. El miembro s5'b está compuesto por conos de escorias y en el sector del Boquerón por andesitas con fenocristales de labradorita, olivino y augita. Por su parte, los depósitos sedimentarios del Cuaternario (Qf) están constituidos principalmente por depósitos coluviales.

\section{MODELO CONCEPTUAL}

El modelo conceptual del sistema acuífero de la cuenca del río San Antonio, se determinó a partir de la interpretación de los sondeos eléctricos verticales (SEV), los cuales se modelaron considerando diversas variables tales como: geología aflorante, niveles estáticos medidos en los pozos, información de columnas litológicas de pozos perforados, pruebas de infiltración y observaciones realizadas en campo.

Para la interpretación, se incluyeron los datos de 15 SEV realizados en el año 2006 y de 40 SEV realizados en el año 1975. El dispositivo eléctrico utilizado fue el Schlumberger y la separación máxima de AB/2 fue de $500 \mathrm{~m}$. El programa de cómputo utilizado para la inversión de los datos fue el QWSELN, el cual realiza la interpretación directa e inversa de los sondeos eléctricos verticales, basada en un modelo tabular o inversión eléctrica en 
Cuadro 1

Resistividad aparente asociada con los materiales identificados en la zona de estudio

\begin{tabular}{lcc}
\hline \multicolumn{1}{c}{ Tipo de material } & Miembro & $\begin{array}{c}\text { Resistividad } \\
(\Omega \mathrm{m})\end{array}$ \\
\hline $\begin{array}{l}\text { Piroclastos y cenizas volcánicas } \\
\text { no saturadas }\end{array}$ & $\mathrm{s} 4$ & $100-150$ \\
$\begin{array}{l}\text { Piroclastos y cenizas volcánicas } \\
\text { saturadas }\end{array}$ & $\mathrm{s} 4$ & $15-50$ \\
$\begin{array}{l}\text { Tobas } \\
\begin{array}{l}\text { Efusivas básicas intermedias no } \\
\text { saturadas }\end{array}\end{array}$ & $\mathrm{s} 3$ 'a & $20-50$ \\
$\begin{array}{l}\text { Efusivas básicas intermedias } \\
\text { saturadas }\end{array}$ & $\mathrm{s} 2$ & $500-1000$ \\
$\begin{array}{l}\text { Efusivas andesíticas y } \\
\text { basálticas }\end{array}$ & $\mathrm{c} 3$ & $70-120$ \\
$\begin{array}{l}\text { Piroclastos ácidos } \\
\text { Piroclastos ácidos saturados }\end{array}$ & $\mathrm{c} 1$ & $100-150$ \\
$\begin{array}{l}\text { Epiclastitas y piroclastitas, lo- } \\
\text { calmente efusivas }\end{array}$ & $\mathrm{b} 1$ & $20-50$ \\
\hline
\end{tabular}

una dimensión. El cuadro 1 muestra los rangos de resistividad aparente asociados con los materiales identificados en la zona de estudio.

Información sobre el fundamento teóricofísico de los métodos geoeléctricos, así como de los diversos dispositivos existentes y de algunas aplicaciones, puede referirse a Orellana (1972), Cantos (1987), Telford et al. (1990), Reynolds (1997), Olmo \& López (1999) y Arias (1999; 2002a; 2002b), entre otros.

A partir del procesamiento, integración e interpretación de los datos existentes, se proponen dos acuíferos, cuyos nombres están basados en la clasificación propuesta por la ASTM (2004).

El acuífero en depósitos piroclásticos del Cuaternario es somero y libre, desarrollado en los miembros s4 y s3'a de la formación San Salvador y en el miembro c1 de la formación Cuscatlán. Los límites del acuífero se determinaron considerando las características geológicas, topografía e inventario de pozos y manantiales. El acuífero presenta un espesor variable; en la zona central de la cuenca, la capa de piroclastos alcanza un espesor medio de $30 \mathrm{~m}$. La dirección preferencial del flujo subterráneo es con orientación noreste, cambiando a sureste en la zona de la desembocadura del río San Antonio (Fig. 3). Las fallas con orientación NW actúan como sectores efluentes del acuífero, pues en el trayecto de $3 \mathrm{~km}$ que existe entre ellas hay un incremento del caudal del río San Antonio de $10 \mathrm{~L} / \mathrm{s}$; además, se aprecia una evidente variación en el gradiente hidráulico en las vecindades de las fallas, lo cual corrobora la influencia de estas estructuras en el acuífero.

El acuífero en lavas fracturadas del Cuaternario se encuentra en el miembro s2 de la formación San Salvador. Su espesor es variable y decrece a medida que desciende del volcán. En algunos sectores y a partir de la correlación litológica de pozos perforados, se estima que su espesor puede alcanzar hasta los $180 \mathrm{~m}$. Hidráulicamente, se comporta preferentemente como un acuífero libre, sin embargo; en el sector central del municipio de Nejapa, los coeficientes de almacenamiento de los pozos perforados en la zona varían entre 1,6

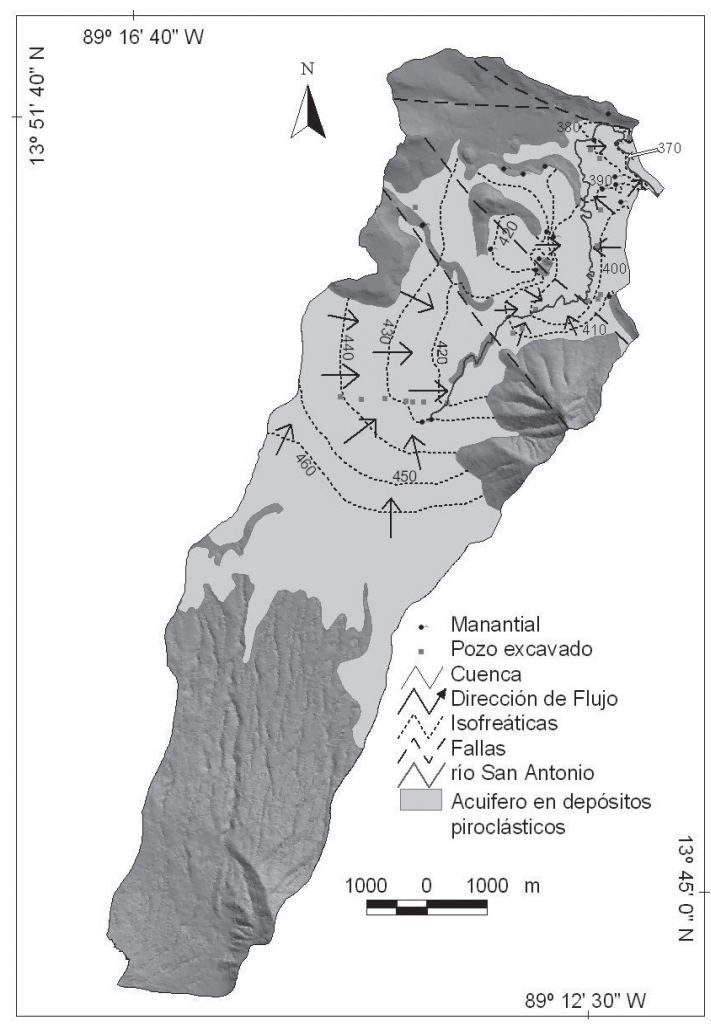

Fig. 3: Mapa de isofreáticas del acuífero en depósitos piroclásticos del Cuaternario. 

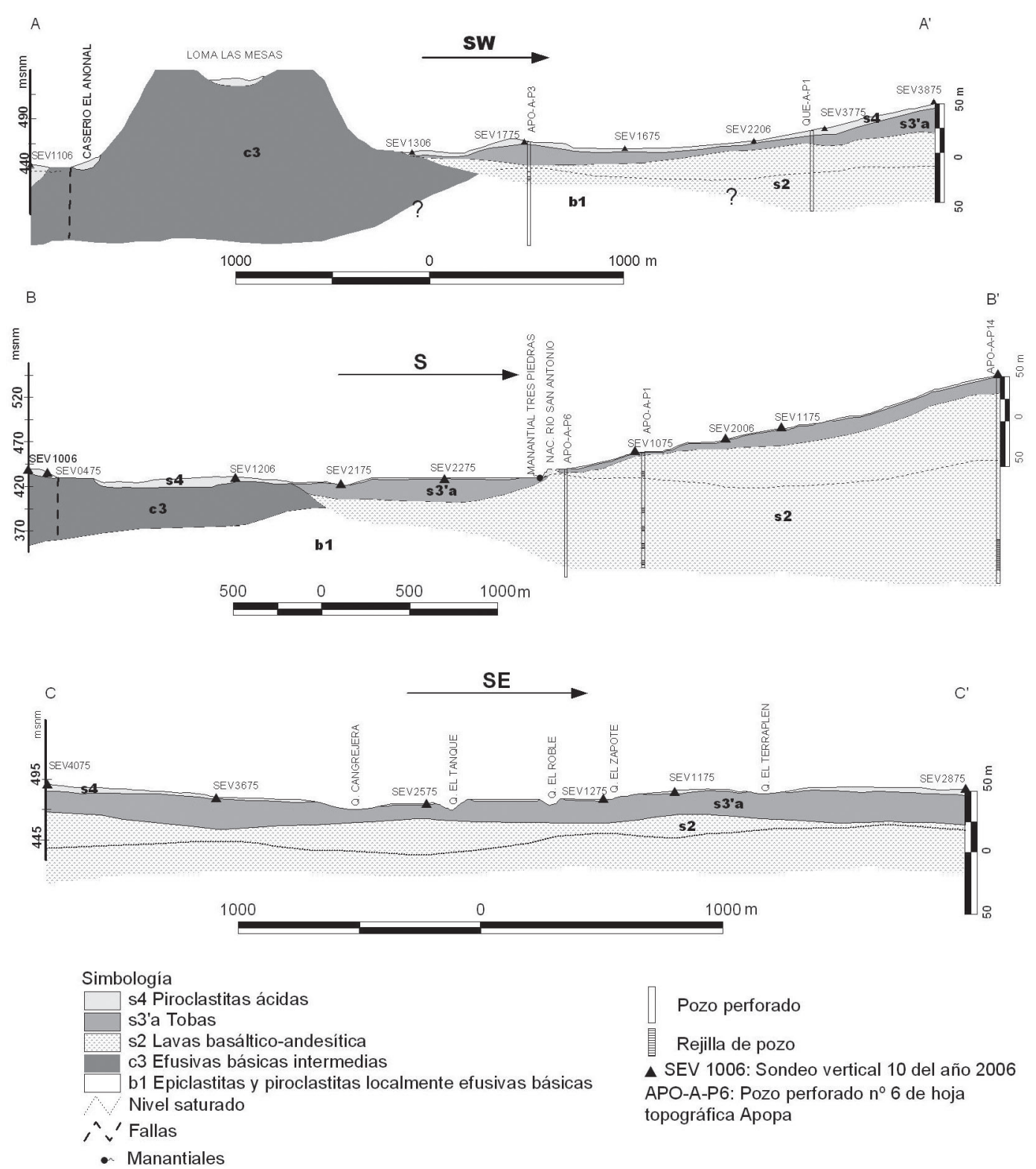

Fig. 4: Perfiles de correlación geoeléctrica, estratigráfica e hidrogeológica.

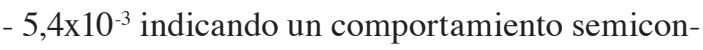
finado (Freeze \& Cherry, 1979). Estos cambios se relacionan con capas de arcilla descritas en las columnas litológicas de los pozos perforados e identificadas como capas conductoras en varios SEV realizados en la zona. El modelo hidrogeológico conceptual contempla la zona de recarga de este acuífero en lavas fracturadas y aflorantes en la parte alta del volcán de San Salvador y debido a la dirección preferencial de flujo, el río San Antonio se convierte en un efluente de este acuífero. Este comportamiento hidráulico se evidenció a partir de aforos diferenciales realizados a lo largo del río y que muestran un incremento de caudal.

Con el fin de caracterizar la distribución espacial de los diversos miembros geológicos que 
albergan a los acuíferos mencionados anteriormente, se describen tres perfiles de correlación geoeléctrica, estratigráfica e hidrogeológica realizados en la cuenca (Fig. 4), cuya localización se ha referenciado en la figura 2 y rangos de resistividad en el cuadro 1 .

El perfil A-A' fue realizado con rumbo SW. En el sector norte de las lomas Las Mesas se encuentra el caserío El Anonal, donde existen manantiales y pozos excavados en los cuales el nivel estático se encuentra aproximadamente a $7 \mathrm{~m}$ de profundidad y asociados al miembro $\mathrm{s} 4$ de la formación San Salvador. Hacia el suroeste de dicha loma afloran también los piroclastos del miembro s4 y subyaciéndolos se encuentran las tobas del miembro s3'a, siendo en este último miembro donde se encuentra el nivel estático del acuífero en depósitos piroclásticos del Cuaternario. El acuífero desarrollado en las lavas fracturadas del Cuaternario se encuentra en el miembro s2, cuyo espesor se incrementa al SW por estar más cerca del volcán de San Salvador. De acuerdo con los sondeos eléctricos verticales realizados y las columnas litológicas de pozos perforados en el sitio, se ha encontrado en estas lavas una zona no saturada de espesor variable entre 20 y $80 \mathrm{~m}$, caracterizada por el valor de resistividad que varía entre 400 - 1000 $\Omega \mathrm{m}$; subyaciéndola se encuentra la zona saturada con un espesor mínimo en este perfil de $5 \mathrm{~m}$ y valor de resistividad del orden de $80 \Omega \mathrm{m}$. Este nivel estático ha sido correlacionado con la información de pozos perforados y es totalmente congruente con ellos.

En el perfil B-B', realizado con rumbo S, el miembro c3 se encuentra aflorando en el sector norte y su espesor va disminuyendo hacia el sur, donde la capa de tobas del miembro s3'a comienza a aparecer, incrementando su espesor hasta llegar al máximo de $40 \mathrm{~m}$ en la zona cercana al pozo APO-A-P6. En el acuífero de depósitos piroclásticos cuaternarios, existe un nivel colgado de agua subterránea con una profundidad media de $3 \mathrm{~m}$. A partir de la dirección de flujo del acuífero desarrollado en lavas fracturadas (de $\mathrm{S}$ a N), de los aforos diferenciales realizados en el sector del nacimiento del río San Antonio (caudal de $53 \mathrm{~L} / \mathrm{s}$ ) y del manantial Tres Piedras (caudal de $228 \mathrm{~L} / \mathrm{s}$ ), así como de la correlación geológica y geoeléctrica, se establece que el acuífero lávico recarga lateralmente (al menos en este sector) al acuífero desarrollado en depósitos piroclásticos, tal como se observa en la figura $3 b$, donde existe una comunicación hidráulica entre los miembros s2 y s3'a. Así, el nacimiento del río San Antonio y el manantial Tres Piedras se originan por las características efluentes del acuífero lávico; además, los pozos perforados en este sector captan también el acuífero desarrollado en lavas fracturadas del Cuaternario.

El perfil C-C', presenta un rumbo hacia el E- SE y cruza transversalmente la parte central de la cuenca, donde la elevación media del terreno es de 500 m s.n.m. En él se observa una distribución horizontal y uniforme de las capas que representan a los diferentes miembros estratigráficos. En superficie y con espesores muy pequeños (entre 3 y $6 \mathrm{~m}$ ), aflora la "Tierra Blanca" (piroclastitas del miembro s4), mientras que en los sectores correspondientes a los cauces de los ríos afloran las "Tobas Café" del miembro s3'a. La base del perfil está constituida por lavas del miembro s2, en las cuales se ha diferenciado a partir de la interpretación hidrogeofísica la zona no saturada con un espesor medio de $20 \mathrm{~m}$ y la zona saturada del acuífero en lavas fracturadas del Cuaternario. Además, en este sector de la cuenca existen pozos perforados que están fuera de uso y que presentan características asociadas al semiconfinamiento, evidenciadas por la surgencia de agua.

\section{VULNERABILIDAD INTRÍNSECA}

La evaluación de la vulnerabilidad intrínseca se realizó únicamente en el sector del acuífero de Depósitos Piroclásticos del Cuaternario donde existe información de pozos y donde se realizaron sondeos eléctricos verticales.

El método GOD desarrollado por Foster \& Hirata (1991), se basa la evaluación de tres parámetros: Tipo de ocurrencia del agua subterránea (G), características de la cobertura del acuífero (O) y profundidad del agua (D). A cada parámetro se le asigna un índice entre 0 (valor mínimo) y 1 (valor máximo), los cuales se multiplican entre sí, obteniendo el índice de vulnerabilidad intrínseca (Fig. 5). 


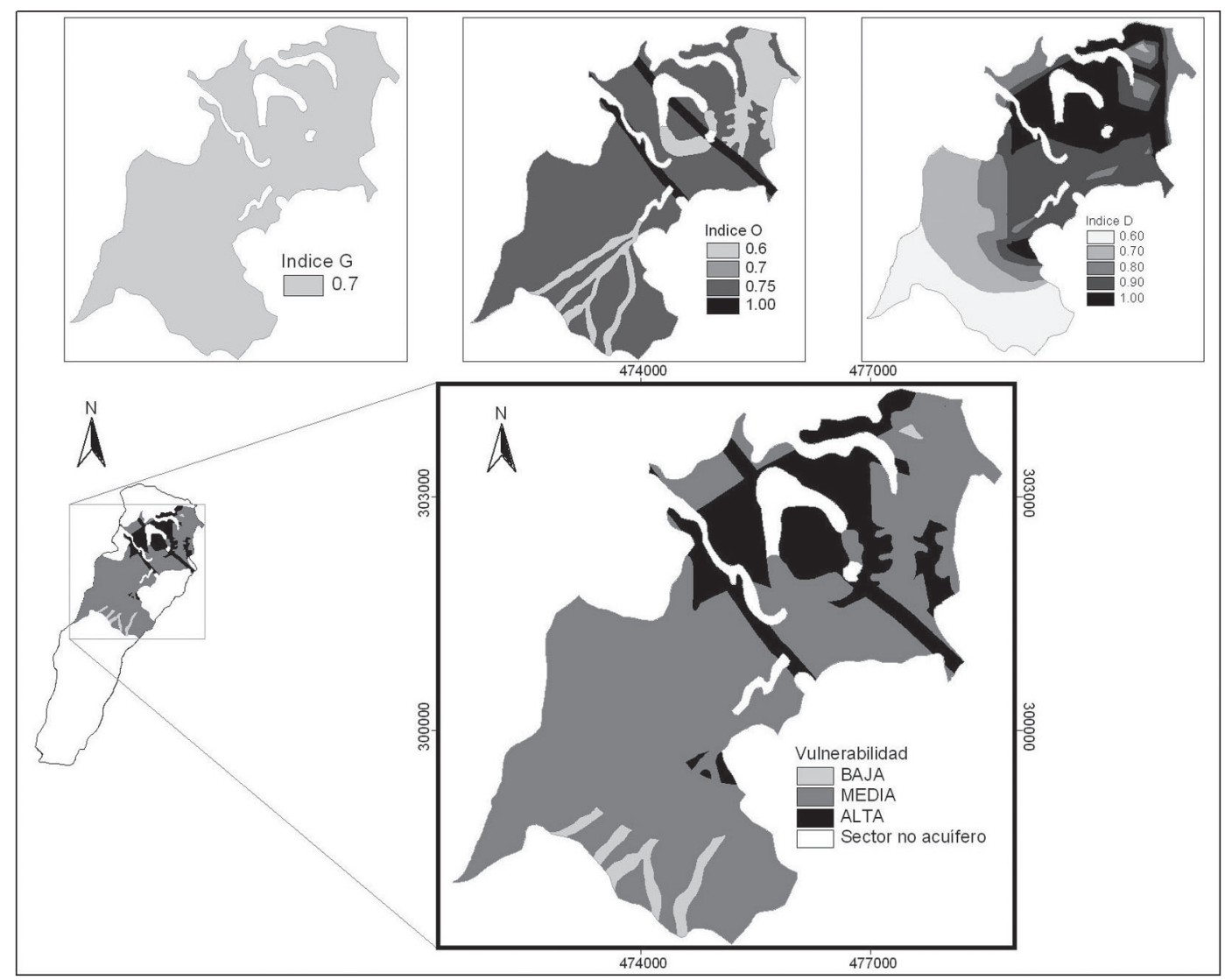

Fig. 5: Mapa de vulnerabilidad intrínseca en un sector del acuífero en depósitos piroclásticos del Cuaternario.

Para determinar la distribución de cada uno de los parámetros, se tomó como base la información de los registros de 21 pozos excavados, 21 manantiales y de 46 sondeos eléctricos verticales, pues con ellos se pudo completar el modelo hidrogeológico conceptual del sitio.

El tipo de ocurrencia del agua subterránea (G): el acuífero somero se clasifica como no confinado. A partir de las valoraciones hechas en el campo, así como de sus características intrínsecas, se le asignó un valor índice de 0,7 en todo el sector analizado.

Cobertura del acuífero $(\mathrm{O})$ : el tipo de material que sobreyace el acuífero es un factor para determinar el grado de vulnerabilidad. La delimitación de este parámetro se basó en la litología, textura y grado de consolidación. El sector del acuífero donde afloran los materiales del miembro c1, se caracterizó con un índice de 0,6; mientras que a la zona cubierta por el miembro s3'a se le asignó un valor de 0,7 . Por su parte, al sector aflorante de "Tierra Blanca" se le estableció un índice de 0,75 . Existen dos fallas que condicionan las características influentes del río San Antonio, ante esta situación se asumió la condición más desfavorable para el acuífero y para brindar el margen de seguridad mayor se le asignó un índice de 1,0 a la zona delimitada a $100 \mathrm{~m}$ a ambos lados de la línea de falla.

Profundidad del agua (D): a partir de una interpolación lineal de los niveles de agua medidos en los pozos y de los niveles aflorantes en los manantiales, se realizó un mapa de profundidad relativa del nivel estático del acuífero que se correlacionó bien con la interpretación hidrogeofísica obtenida de los SEV. Los índices asignados están en función de los rangos establecidos en la metodología GOD y varían entre 0,6 y 1,0. 
Vulnerabilidad del acuífero: el acuífero somero presenta una vulnerabilidad baja en un $3 \%$ de su extensión, misma que se ha asociado con el afloramiento de tobas del miembro s3'a. Por su parte, la vulnerabilidad media es la que predomina en casi toda su extensión (77\%). En la zona noroeste del acuífero y en las zonas aledañas a las fallas se observan índices de vulnerabilidad altas representando un 20\% del área evaluada. La alta vulnerabilidad está asociada con niveles estáticos someros (menores a $2 \mathrm{~m}$ ) que inciden en la asignación de valor máximo $(\mathrm{D}=1)$ en un sector importante del acuífero, así como a la existencia de fallas que condicionan el valor máximo en el parámetro cobertura de acuífero $(\mathrm{O}=1)$.

\section{CONCLUSIONES}

La caracterización hidrogeofísica de la cuenca del río San Antonio, realizada a partir de la interpretación conjunta de sondeos eléctricos verticales con la información litológica de pozos, la correlación geológica de mapas previos y de las observaciones y ensayos de campo, ha permitido diferenciar dos unidades acuíferas.

El acuífero en depósitos piroclásticos del Cuaternario, está constituido por los miembros s4 y s3'a de la formación San Salvador y el miembro c1 de la formación Cuscatlán. En la actualidad el uso primordial de este acuífero es para uso doméstico por medio de pozos excavados artesanalmente y captación de manantiales.

El miembro s2 de la formación San Salvador, alberga al acuífero de lavas fracturadas del Cuaternario, que es explotado por varios pozos perforados (privados y públicos) para abastecimiento público de los habitantes del municipio de Nejapa y lugares cercanos.

La aplicación e interpretación de los sondeos eléctricos verticales permitió diferenciar con cierto grado de precisión el espesor y/o profundidad de los diferentes miembros de las formaciones geológicas existentes en la zona de estudio, siendo este un aporte importante para determinar la distribución espacial de las unidades estratigráficas y así poder plantear un modelo hidrogeológico conceptual que ha permitido la determinación del índice de vulnerabilidad intrínseca de un sector del acuífero desarrollado en depósitos piroclásticos del Cuaternario.

Las características hidrogeológicas, la profundidad del nivel del agua y la cobertura del acuífero en depósitos piroclásticos, evidencian una vulnerabilidad baja en un 3\% del área evaluada, la vulnerabilidad media para el área considerada es de $77 \%$ y una vulnerabilidad alta está presente en un $20 \%$ del área. Por esto, es imperativo que las instituciones públicas de El Salvador tomen conciencia de estas características y promuevan un ordenamiento sostenible del uso de la tierra, que esté acorde con la protección del recurso hídrico. Así, las actividades urbanística, agrícola, ganadero e industrial en las áreas de vulnerabilidad baja y media del acuífero somero, deberían contemplar medidas de control. Específicamente en los referente a sistemas de eliminación de aguas servidas y tratamiento de efluentes en general, la densidad de población, el área de los lotes de tal manera que el área de impermeabilización por hectárea no exceda un $30 \%$ del espacio, utilización de agroquímicos de muy baja toxicidad, persistencia y movilidad, así como prácticas apropiadas para la conservación de suelos. Por su parte, en los sectores de alta vulnerabilidad, deberán incrementarse las restricciones en el uso del suelo, solicitar estudios hidrogeológicos específicos y prohibir algunas actividades con alto riesgo de contaminación, relacionadas con el tratamiento, disposición y manejo de sustancias tóxicas y efluentes de procesos industriales.

\section{AGRADECIMIENTOS}

Por el aporte de financiamiento parcial y técnico-logístico, los autores desean agradecer a la Agencia Alemana de Intercambio Académico (DAAD), la Red Centroamericana de Manejo de Recursos Hídricos (CARA), la Administración Nacional de Acueductos y Alcantarillados (ANDA), la Universidad de El Salvador (UES), la Universidad Centroamericana José Simeón Cañas (UCA) y la Universidad de Costa Rica (UCR). 


\section{REFERENCIAS}

ASTM, 2004: Standard Guide for Establishing Nomenclature of GroundWater Aquifers.17 págs. ASTM Intenational, West Conshohocken.

ARÉVALO, D., 2005: Evaluación de la vulnerabilidad intrínseca del acuífero OpicoQuezaltepeque, a través de datos geofísicos, pozos y análisis hidrogeoquímicos, El Salvador, Centro América.- 76 págs. Univ. Costa Rica, San José [Tesis M.Sc.]

ARIAS, M. E., 1999: Les méthodes de sondages électriques et électromagnétiques: Examples d'application en Hydrogéologie.- 92 págs. Université Pierre et Marie Curie, Paris. [Inf. DEES].

ARIAS, M. E., 2002a: Aplicaciones geofísicas a la hidrogeología de Costa Rica.- Rev. Geól. Amér. Central, 27: 11-20.

ARIAS, M. E., 2002b: La prospección geoeléctrica y electromagnética en dos dimensiones y su aplicación en la hidrogeología.Rev. Geól. Amér. Central, 27: 21-26.

BAXTER, S., 1984: Léxico estratigráfico de El Salvador.- 108 págs. Comisión Ejecutiva Hidroeléctrica del río Lempa (CEL), San Salvador.

CANTOS, J., 1987: Tratado de Geofísica Aplicada.- 535 págs. Promotora de Minas de Carbón, Madrid.
FOSTER, S. \& HIRATA, R., 1991: Determinación del riesgo de contaminación de agua subterránea.- 80 págs. Centro Paramericano de Ingeniería Sanitaria y Ciencias del Ambiente, $2^{a}$ edición, Lima.

FREEZE, R.A. \& CHERRY, J.A., 1979: Groundwater.- 604 págs. Prentice Hall. Englewood Cliffs, New Jersey.

MISIÓN GEOLÓGICA ALEMANA, 1978: 6 mapas geológicos de la República de El Salvador.- Escala 1:100 000. Bund. F. Geow. U. Rohs. Hannover.

OLMO, M. \& LÓPEZ, J. (eds.) 1999: Actualidad de las técnicas geofísicas.- 375 págs. Instituto Tecnológico Geominero de España, Madrid.

ORELLANA, E., 1972: Prospección geoeléctrica en corriente continua.- 523 págs. Paraninfo, Madrid.

REYNOLDS, J., 1997: An Introduction to Applied and Environmental Geophysics.- 796 págs. Wyley, London.

TELFOR, W., GELDART, L. \& SHERIFF, R., 1990: Applied Geophysics.- 770 págs. Cambridge University Press, London.

WIESEMANN, G., 1975: Remarks on the geologic structure of the republic of El Salvador, Central América.- Mitt. Geol.Paläont. Inst.: 557-573. 
